\title{
DESIGN YOUR OWN LAB EXPERIMENTS: PROJECT-BASED LEARNING APPROACH TO TEACHING CHEMICAL PRINCIPLES OF ENGINEERING
}

\author{
Gorka Gallastegui, Juan Jesús López, Jon Alvarez, Arrate Santaolalla, \\ \& Junkal Gutierrez \\ Department of Chemical and Environmental Engineering, Faculty of Engineering Vitoria-Gasteiz, \\ University of the Basque Country (Spain)
}

\begin{abstract}
This study introduces the substitution of traditional laboratory practicals related to acid-base titrations and reduction-oxidation reactions carried out to date (where students only have to repeat the instructions provided by the teacher in a manual or script) by new ones where students are responsible for planning and conducting a small research project within the framework established by the teaching staff. Thus, each group of students develops an experimental design regarding topics such as, fabrication of an alcoholmeter, determination of the oxidative capacity of household cleaners or preparation of acid-base indicators based on natural products.

The promise of finding a real solution for a daily challenge becomes the motivation for learning. Therefore, the aim of the proposed methodology is focused in achieving greater involvement of students, as well as effective knowledge acquisition. Similarly, it is intended to promote students' initiative and expand their creativity.
\end{abstract}

Keywords: Active learning pedagogy, chemical education, laboratory experiments, problem-based learning.

\section{Introduction}

Present-day society is characterized by the free access to a seemly endless amount of dynamic and changing information, the massive use of the Internet, and above all, a constantly evolving job market. Consequently, future engineers should excel, not only because of their specific and vast knowledge base, but also due to their "soft skills". These desirable qualities do not depend on acquired knowledge, but they allow complex problems to be resolved in multidisciplinary groups by means of an efficient and creative approach. For current engineering students, the challenge in adapting to this new reality will be to develop the capacity for self-directed learning throughout their whole working live (Dochy et al., 2005).

As a result, the implementation of the European Higher Education Area (EHEA) in 2010 following the Bologna Declaration meant the transformation of the studies related to Industrial Engineering specialties in Spain (European Higher Education Area [EHEA], 2016). The new teaching/learning paradigms focus on self-directed learning, which facilitate the ongoing process of developing, maintaining and documenting the professional skills, and the conversion of students into the main actors of their own learning process (Rodríguez Izquierdo, 2014). With the goal of helping students to become enthusiastic life-long learners, several actions should be taken into account by Universities:

- Adapt the work completed by the students (i.e., hours of class-time, written work and practicals, and individual-study) to the European Credit Transfer System (ECTS) structure.

- Design new study programs based on professional and academic profiles that address the aforementioned needs of the job market.

- Encourage student to take a more active role in their learning.

However, traditional lecture-based instruction is still predominant in many Spanish Universities, making it more difficult to achieve valuable skills such as the ability to learn, to organize and to plan, or to adapt to new situations. In order to turn the scenario around, EHEA recommends the implementation of active learning methodologies as the principal approach to teach students to learn how to learn. In an active learning classroom, students must think, create and solve problems rather than passively listen to lecture. In summary, it is the student who constructs knowledge following guidelines, activities or scenarios designed by the teaching staff. 


\section{Objective and conceptual framework}

In this work, an alternative didactic approach based on active pedagogies is proposed in order to conduct laboratory practicals within the subject of Chemical Principles of Engineering, a compulsory first-year subject for students of Industrial Engineering specialties (i.e., Mechanical Engineering, Industrial Electronic Engineering, and Industrial Chemical Engineering) at the Faculty of Engineering Vitoria-Gasteiz (University of the Basque Country (UPV/EHU)).

Chemical Principles of Engineering (9 ECTS) is devoted to providing an understanding of knowledge regarding structure, properties and behaviour of matter, as well as a deep comprehension of certain industrial processes. The course curriculum is covered in 45 lecture hours and 15 classroom practical hours. Outside of class, after basic knowledge acquisition during the first quarter of the course, students perform five $3 \mathrm{~h}$ laboratory sessions in the $2^{\text {nd }}$ quarter, which are individually evaluated by a multiple-choice test filled out at the end of each session and a written laboratory report. Laboratory practicals are considered a fundamental activity both to learn basic experimental techniques typically used within a chemistry lab and to attain the practical skills that graduates with a Bachelor's Degree in Industrial Engineering should demonstrate on a daily basis in their future profession.

\section{Design and methodology}

In an attempt to engage students in the classroom, real world applications related to course material are incorporated into the laboratory practical devoted to acid-base titrations and reduction-oxidation reactions. Thus, students are responsible for planning and conducting a small research project within one of the following frameworks (Figure 1):

1. Configuration of galvanic cells: Daniel cell and its construction based on natural products (e.g., fruits, liquids, etc.). Nernst equation.

2. Fabrication of an alcoholmeter (determination of alcohol concentration in gaseous media).

3. Determination of the oxidative capacity of household cleaners (e.g., blench).

4. Understanding the acid neutralizing mechanism of commercial antacids.

5. Linking wine quality with acid-base balances and precipitation reactions.

6. Preparation of acid-base indicators based on natural products (e.g., purple cabbage or spinach) and determination the acidity of the soil from the University garden.

Figure 1. Covers of the daily challenges proposed to the students.

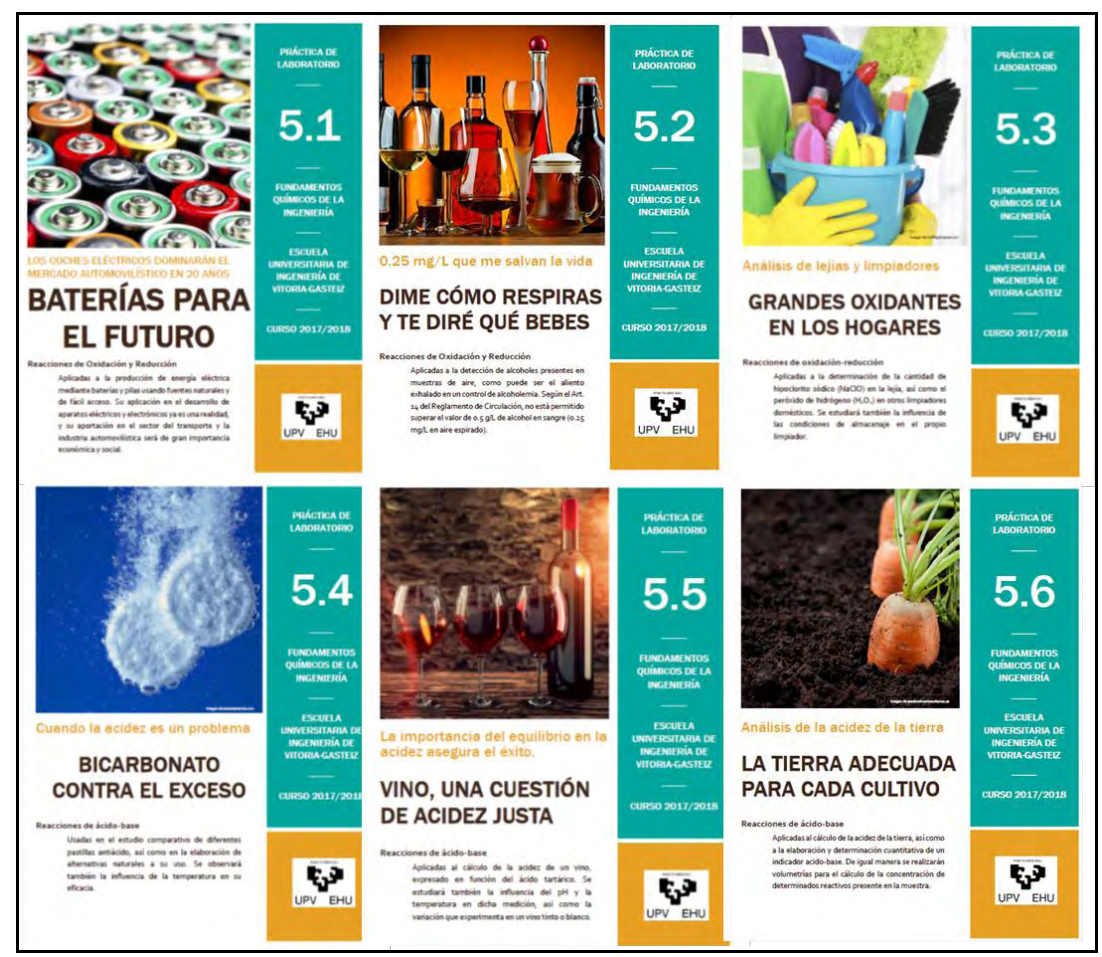


These small research projects are developed according to problem-based methodology (PBM). This method gives students the opportunity to highlight real-life applications of course content and it is intended to be an interesting challenge to demonstrate their chemical knowledge. As such, the activity is implemented using the following guidelines:

- Presentation of daily challenges: teaching staff must present some attractive research topics to bring chemistry closer to students.

- Articulation of the studies' boundaries or limitations: it should be taking into account that students could be swamped and feel overwhelmed due to their limited knowledges of chemistry. (Give students some tips: brief theoretical explanation, news appeared in the press, different approaches, etc.)

- Revision of the student proposal according to resources available in the laboratory of the University. Research protocol realized by students require an initial assessment to ensure the feasibility of the proposal (check chemical reagents, lab facilities and resources).

- Project Implementation: students check self-designed protocol in the laboratory and analyse obtained results:

- Elaboration of a final video report: students have to perform a video as an alternative to traditional paper-report.

\section{Expected results}

Proposed activity allows students to design their own lab experiments by means of project-based learning approach. The strengths of the teaching methodology presented herein are mostly related to the added value given to the learning program: students take a central role in their own learning process and are more motivated since they find pleasure in what they are doing. Regarding the learning outcomes, by the end of the practical, students will be able to:

- Acquire advanced chemistry knowledge by means of contextualized and practical learning.

- Realise the applicability of theoretical knowledge in real cases.

- Increase initiative and creativity levels.

- Improve oral communication skills.

- On the contrary, several threatening clouds on the horizon could doom this proposal to failure:

- Assessment criteria for the practical is much more complex and it could not be directly related to successful achievement of the challenge posed.

- Practical preparation and subsequent monitoring is much more time-consuming in comparison with the classical approach.

- Unreflective copying of inaccurate information on the Internet by the students.

- The requirement to expose new challenges every academic course to avoid any plagiarism.

\section{References}

Dochy, F., Segers, M., van den Bossche, P., \& Gijbels, D. (2005). Effects of problem-based learning: a metaanalysis. Learning and Instruction, 13, 533-568.

European Higher Education Area. (2016). European Higher Education Area and Bologna Process. Retreived April 5, 2019, from http://www.ehea.info/.

Rodríguez-Izquierdo, R.M. (2014). Modelo formativo en el Espacio Europeo de Educación Superior: valoraciones de los estudiantes. Aula Abierta, 42, 106-113. 